\title{
LA "TRIPLE PRESENCIA-AUSENCIA": UNA PROPUESTA PARA EL ESTUDIO DEL TRABAJO DOMÉSTICO-FAMILIAR, EL TRABAJO REMUNERADO Y LA PARTICIPACIÓN SOCIOPOLIITICA
}

\section{The "triple presence-absence": a proposal for the study of domestic-family work, paid work and social and political participation}

\author{
Marina Sagastizabal*1; Matxalen Legarreta** \\ * Universidad del País Vasco/Euskal Herriko Unibertsitatea; ** Universidad del País Vasco/Euskal \\ Herriko Unibertsitatea \\ marina.sagastizabal@ehu.eus; matxalen.legarreta@ehu.eus
}

Palabras clave
Triple presencia-
ausencia
Materialidad
Aprendizaje de
género
Ética del cuidado

Keywords
The triple
presence-absence
Materiality
Doing gender
Ethic of care

Resumen

El artículo tiene como objetivo ofrecer una reflexión sobre la "triple presencia-ausencia", una propuesta para el estudio de los cuidados que parte de una mirada amplia, abordando su relación con el trabajo remunerado y con la participación sociopolítica. La propuesta se sitúa en los debates desarrollados en las últimas décadas en torno a los cuidados, principalmente en relación a tres líneas de trabajo centradas respectivamente en: la dimensión material del trabajo domésticofamiliar, el aprendizaje de género y la ética del cuidado. La potencialidad de la noción la "triple presencia-ausencia" descansa en su capacidad para complejizar el análisis de los cuidados en dos sentidos. Por un lado, relaciona los cuidados no sólo con el trabajo remunerado (como lo hacen planteamientos predecesores en torno a la "doble jornada" o la "doble presencia-ausencia"), sino también con la participación sociopolítica. Por otro lado, presta atención tanto a la materialidad (ocupaciones en sentido estricto) como a los aspectos subjetivos y morales del trabajo domésticofamiliar (responsabilidad, disponibilidad, preocupación...). Es una propuesta de corte eminentemente analítico que deberá ser contrastada a nivel empírico en futuros trabajos.

\begin{abstract}
The article aims to provide a reflection on the "triple presence-absence", a proposal to study care of a broad view, addressing its relationship with paid work and political involvement. The proposal is made in the discussion developed the last few decades around care, mainly related to three working lines focused respectively on: the material dimension of domestic-family work, doing gender effect and ethic of care. The potential of the notion the "triple presence-absence" lies in its ability to make more complex the analysis of care in two ways. On the one hand, it relates care not only with paid work (as do predecessor approaches around the "double shift" or "double presence-absence"), but also with socio-political participation. Furthermore, it pays attention to both the materiality (occupations in the strict sense) as well as to subjective and moral aspects of domestic-family work (responsibility, availability, worry...). It is an eminently analytical proposal, which shall be contrasted empirically in future studies.
\end{abstract}

Sagastizabal, M. y Legarreta, M., 2016, "La "triple presencia-ausencia": una propuesta para el estudio del trabajo doméstico-familiar, el trabajo remunerado y la participación sociopolítica”, en Papeles del CEIC, vol. 2016/1, no 151, CEIC (Centro de Estudios sobre la Identidad Colectiva), Universidad del País Vasco, http://dx.doi.org/10.1387/pceic.15447

\footnotetext{
${ }^{1}$ Marina Sagastizabal tiene una beca FPU del Ministerio de Educación, Cultura y Deporte para la realización de su tesis doctoral. Este artículo se sitúa en el marco de dicha tesis.
} 


\section{INTRODUCCIÓN ${ }^{2}$}

El trabajo doméstico y los cuidados constituyen un ámbito de investigación relativamente reciente en las ciencias sociales, pero que ha sido considerado un "terreno" emblemático para estudiar las desigualdades de género (Hughes, 2002). Las primeras aportaciones en este campo se desarrollan en los años sesenta y setenta del siglo pasado con la finalidad de poner de manifiesto su importancia para la supervivencia y el bienestar de la sociedad, así como las desigualdades de género sobre las que descansa (Borderías, Carrasco y Alemany, 1994). La mayor parte del esfuerzo se dirige a conceptualizar las actividades que se desempeñan en el ámbito doméstico-familiar como trabajo meramente físico, dejando a un lado la parte subjetiva, emocional y moral.

Con el tiempo, los debates ponen el foco de atención precisamente en estos últimos aspectos, considerándolos una parte inherente del cuidado, pues éste involucra a la persona en todas sus capacidades (Anderson, 2000). Con todo, hoy por hoy, se puede afirmar que hay unanimidad a la hora de considerar ambas dimensiones como parte del trabajo doméstico-familiar ${ }^{3}$ (Carrasco, Borderías y Torns, 2011) y el care (cuidado) se ha institucionalizado como concepto y perspectiva analítica a nivel europeo (Letablier, 2007) y en el continente americano.

La propuesta en torno a la "triple presencia-ausencia" nace en este marco. Concretamente, se nutre de tres grandes líneas desarrolladas en torno a los debates sobre la conceptualización y el análisis del trabajo doméstico y los cuidados. La primera, pone el foco de atención en los aspectos materiales; la segunda, en el aprendizaje de género; $y$, la tercera, versa sobre la ética del cuidado. Ahondando en la potencialidad y en los límites de cada una de ellas, aborda el trabajo domésticofamiliar, así como su relación con el trabajo remunerado y la participación sociopolítica. Se trata de una propuesta de corte eminentemente analítico que deberá ser contrastada a nivel empírico en futuros trabajos.

\footnotetext{
${ }^{2}$ Queremos agradecer a Gisela Bianchi-Pernasilici y a las dos evaluadoras anónimas sus sugerencias y su atenta lectura de este texto. No obstante, la responsabilidad última del mismo es únicamente de las autoras.

${ }^{3}$ Empleamos trabajo doméstico-familiar para hacer referencia tanto al trabajo doméstico como a los cuidados.
} 
El artículo se divide en cuatro apartados. Los tres primeros ofrecen una revisión de las citadas líneas de trabajo sobre las que descansa la propuesta para, en el cuarto, presentar la "triple presencia-ausencia" y exponer su potencialidad.

\section{EL PUNTO DE PARTIDA: LOS ASPECTOS MATERIALES DEL TRABAJO}

\subsection{Trabajo, empleo y ciudadanía}

Las sociedades modernas occidentales se han constituido sobre la división de dos esferas: la pública y la privada. Esta separación ha otorgado privilegios a los hombres y subordinado a las mujeres (Moghadam, 2003; Pateman, 1996). De esta forma, la diferencia sexual se ha convertido en un determinante social y simbólico que ha situado a las mujeres en el ámbito de lo privado, de las emociones y de la naturaleza, en contraposición a los hombres, posicionados en lo público, la razón y los derechos (Young, 1990; Benhabib, 1990).

En consecuencia, el pensamiento científico ha dirigido su atención teórica y práctica exclusivamente al ámbito público, dando por supuesto que la vida doméstico-familiar es irrelevante tanto para la teoría social y política como para la economía (Pateman, 1996). Por esta razón, una de las principales preocupaciones de la teoría feminista ha sido poner en relación ambas esferas, señalando la relevancia de la domésticafamiliar, pilar imprescindible para que la esfera productiva-mercantil se sostenga. En este sentido, se han realizado análisis exhaustivos sobre la base material de las actividades realizadas en el hogar, concluyendo que constituyen un trabajo (Carrasquer et al., 1998).

El debate entre el feminismo y el marxismo ha sido esencial para reconocer y dar valor al trabajo que históricamente han llevado a cabo las mujeres en el ámbito doméstico-familiar (Galcerán, 2006). Además, es importante tener en cuenta que la concepción de ciudadanía ha tenido en la categoría social del trabajo (remunerado) el elemento central para su constitución y regulación (Alonso, 2007; Prieto, 2007), lo que ha supuesto que las mujeres hayan sido consideradas ciudadanas "de segunda". Excluir a las mujeres de la categoría "empleo" y, por ende, de los derechos de ciudadanía que se derivan del mismo, ha creado situaciones de desigualdad y exclusión por razón de género (Alonso, 2007). Estas desigualdades se refuerzan a través del "contrato sexual" 
(Pateman, 1995); es decir, el sistema por el cual se supone que en cada hogar existe un "hombre-ganador de pan" que aporta un salario familiar y una "mujer-ama de casa" que procura el bienestar cotidiano necesario (tanto material como afectivo). Éste es un sistema jerárquico del que deriva un reparto de derechos, deberes, espacios, tiempos y funciones desigual que privilegia a los hombres frente a las mujeres.

\subsection{Trabajo, empleo y ciudadanía}

Si bien los trabajos de las historiadoras han visibilizado la contribución de las mujeres al mercado laboral en la época de la industrialización, poniendo de manifiesto que las mujeres de clases populares han desempeñado tradicionalmente trabajos remunerados (Scott, 1982; 1993), cabe afirmar que a partir de los años sesenta del siglo $X X$ en occidente las mujeres se han ido incorporando progresivamente y de forma más generalizada al mercado laboral. Este hecho, en parte, ha modificado el sistema de división sexual anterior (Alcañiz, 2015; Folbre y Nelson, 2000). No obstante, un mayor protagonismo por parte de las mujeres en el ámbito productivo-mercantil, no ha sido correspondido con una implicación equivalente por parte de los hombres en el trabajo doméstico-familiar (Gershuny y Robinson, 1988). De esta forma, desde el feminismo se propone la noción "doble jornada" (Hochschild y Machung, 2003; Durán, 1986) para visibilizar que las mujeres, lejos de dejar de lado sus "funciones domésticas" (Murillo, 1996), las han sumado al trabajo en el ámbito laboral. Esta situación está caracterizada por trayectorias laborales precarias, instauradas en la temporalidad, la parcialidad y las entradas y salidas del mercado laboral, derivadas de la asunción de las ocupaciones y responsabilidades doméstico-familiares (Cordoni, 1993; Carrasquer, 2002).

Desde esta perspectiva, nace también el término "carga global de trabajo", una categoría que hace referencia "a la suma de trabajo remunerado y no remunerado observada desde la perspectiva individual y desde la suma total" (García Sainz, 2002: 237). El trabajo se considera, así, como un todo en el que ambas partes, mercado y no mercado, están interrelacionadas. De esta forma, se sostiene que para entender lo que acontece en el mercado resulta imprescindible dar cuenta de lo que sucede en los hogares, y viceversa, para tener una información más fehaciente de las dinámicas de los hogares se recomienda mirar a lo que ocurre en el mercado. Desde esta mirada, es posible sacar a la luz los 
condicionantes externos de la participación de las mujeres en el trabajo: la dedicación al mercado laboral viene determinada por su implicación en el ámbito doméstico-familiar, del mismo modo que una desigual distribución entre mujeres y hombres del trabajo doméstico y los cuidados conlleva un desigual acceso y participación por parte de éstas en el mercado laboral.

A nivel analítico, a través de esta noción es posible estudiar el trabajo desde una perspectiva triple. Por un lado, se puede conocer cómo se distribuye en una determinada sociedad el trabajo remunerado y el no remunerado, a saber, quién realiza cada actividad: ¿existe una participación desigual de mujeres y hombres en cada ocupación? Por otro lado, cabe determinar sobre qué tipo de trabajo se mantiene la sociedad: ¿en la sociedad en su conjunto, tiene mayor peso el trabajo remunerado o el no remunerado? Y por último, tomar en consideración tanto las actividades remuneradas como las no remuneradas ofrece la posibilidad de identificar si existe una desigual distribución del trabajo en función del género: ¿en general, quién realiza una mayor cantidad de trabajo, las mujeres o los hombres?

En este sentido, tomando en cuenta la carga global de trabajo a nivel individual, el reciente análisis de los datos producidos a través de la Encuesta de Presupuestos de Tiempo de Eustat (1993-2013) muestra cómo entre 1998 y 2003 (años de bonanza económica en el contexto vasco y español), los hombres de la Comunidad Autónoma de Euskadi por cada hora dedicada al ámbito doméstico-familiar, emplean aproximadamente cinco horas al trabajo remunerado (cinco veces más ellos que ellas), lo que pone en evidencia la persistencia de su rol de principal sustentador del salario familiar o de "hombre-ganador de pan" ${ }^{\prime 4}$. Es más, cabe destacar que en comparación con otros países

\footnotetext{
${ }^{4}$ Datos obtenido de los resultados de la Encuesta de Presupuestos de Tiempo de Eustat (1993-2013) disponibles en:

http://www.eustat.eus/estadisticas/tema_173/opt_0/temas.html - axzz3s/2pQcko. Última consulta: 23/11/2015. Cabe señalar la existencia de diferencias en los comportamientos de mujeres y hombres según la edad o la participación en el mercado laboral. Así, por ejemplo, en general la población joven emplea menos tiempo que la población adulta y mayor en el trabajo doméstico y los cuidados; entre las mujeres y los hombres, aquellas que están fuera del mercado laboral, sin ser estudiantes, dedican al ámbito doméstico y los cuidados más tiempo que los que trabajan de forma remunerada, aunque las mujeres con empleo invierten más tiempo que los hombres desempleados y jubilados. Se ha llevado a cabo un análisis más exhaustivo en este sentido en trabajos anteriores (Sagastizabal y Luxán, 2015; Legarreta y García Sainz, 2015).
} 
europeos y con el Estado español en su conjunto, para el periodo en torno al año 2000, la Comunidad Autónoma de Euskadi es la región en la que se reproduce el modelo "hombre-ganador de pan" de forma más acusada, pues es donde para ellos cobra mayor peso el trabajo remunerado: $75 \%$ de su carga global de trabajo, siendo este porcentaje menor en el resto de países ${ }^{5}$ (Legarreta y García Díez, 2015). Por el contrario, en el caso de las mujeres, entre 1993 y 2013, por cada hora dedicada al ámbito doméstico-familiar, destinan poco más de una hora al trabajo remunerado, por lo que se puede afirmar que ellas han sido y siguen siendo las principales responsables de la conciliación de la vida laboral y familiar 6 .

La noción carga global de trabajo se hace operativa en todas sus dimensiones cuando al trabajo doméstico-familiar y al remunerado se le suma el cómputo de tiempo empleado en el voluntariado y en la participación sociopolítica. De esta forma, nace la propuesta "carga total de trabajo" (Moreno y Borràs, 2013) con el fin de introducir en el análisis la parte del trabajo no remunerado que no se ciñe a lo domésticofamiliar. De esta forma, en el contexto de las Encuestas de Presupuestos de Tiempo la distribución de la carga de trabajo es un indicador fundamental de la "doble jornada", que se vuelve "triple", si tomamos en cuenta todos los trabajos necesarios para el sostenimiento de la vida, pues como apunta Bryson (2007): ¿acaso cuando alguien ayuda a sus vecinas y vecinos o participa en una asociación, no está realizando un trabajo?

En este sentido, Taylor (2005) advierte que el trabajo voluntario ha sido ignorado por parte de la sociología del trabajo. No obstante, ella lo define esencialmente como el trabajo no pagado realizado en el ámbito

\footnotetext{
${ }^{5}$ A nivel europeo sólo hay datos armonizados para el periodo en torno al año 2000 (entre 1998 y 2006), por lo que las comparaciones entre países únicamente se pueden llevar a cabo en relación a esta fecha. Los países europeos que han proporcionado datos sobre el empleo del tiempo son: Bélgica, Bulgaria, Alemania, Estonia, España, Francia, Italia, Letonia, Lituania, Polonia, Eslovenia, Finlandia, Reino Unido y Noruega. Están disponibles en Eurostat: http://ec.europa.eu/eurostat/cache/metadata/en/tus_esms.htm. Los datos de Eustat son los producidos a través de la Encuesta de Presupuestos de Tiempo de 2003. Se realiza una comparación más exhaustiva del contexto europeo en un trabajo anterior (Legarreta y García Sainz, 2015).

${ }^{6}$ Datos obtenido de los resultados de la Encuesta de Presupuestos de Tiempo de Eustat (1993-2013) disponibles

en:

http://www.eustat.eus/estadisticas/tema_173/opt_0/temas.html - axzz3s/2pQck.

Última consulta: 23/11/2015.
} 
público, rompiendo así la dicotomía entre lo público/pagado y lo privado/no pagado. A partir de un estudio realizado en Gran Bretaña, esta autora matiza que no todas las personas que realizan este trabajo se identifican como "voluntarios/as" y, por ello, ve necesario vincularlo al contexto y a las experiencias relacionadas con el propio trabajo. En este sentido, señala que la definición de "voluntariado" históricamente ha tenido un sesgo de clase importante: se ha relacionado con actividades caritativas realizadas por personas blancas y de clase media (a menudo mujeres). Sin embargo, a pesar de que dentro del movimiento obrero se llevan a cabo a menudo trabajos voluntarios similares, éstos no se identifican con la figura del "voluntario/a". Así, mientras que la experiencia del voluntariado de clase media se relaciona con situaciones de poder asimétricas, privilegios y respetabilidad (o estatus), en el contexto de las clases populares se guía por relaciones de solidaridad, reciprocidad y apoyo comunitario (Taylor, 2005).

Gracias a las aportaciones de Taylor $(2004,2005)$ la división entre las esferas pública y privada se complejiza y cobra un interés especial en este terreno. Así, se aboga por reconceptualizar el trabajo desde una mirada que abarque el ámbito de la participación sociopolítica.

\subsection{Aportaciones y límites de atender a la materialidad}

Una mirada a la distribución del tiempo diario desde la perspectiva de la carga total de trabajo pone de manifiesto que las mujeres trabajan más horas que los hombres, lo que se relaciona directamente con la "pobreza de tiempo" (Bryson, 2007; Sayer, 2005) experimentada principalmente por parte de ellas. Así, en los veinte años que abarca el estudio de las Encuestas de Presupuestos de Tiempo de Eustat, los hombres han disfrutado de un mayor volumen de tiempo de libre disposición en comparación con las mujeres ( 5 horas y 31 minutos diarios de promedio ellos, y 4 horas y 42 minutos ellas). Además, en todo el periodo, por cada hora de trabajo, las mujeres no han llegado a disponer de 45 minutos de tiempo para emplearlo libremente (Sagastizabal y Luxán, 2015). Disponer de una mayor cantidad de tiempo libre conlleva ventajas a la hora de estar presente en ámbitos como el laboral, el social o el político (Bryson, 2007, 2008). Además, el tiempo libre de ellas es menos "utilizable" que el de ellos, ya que a menudo aparece "contaminado" por el cuidado (Bryson, 2007) y se identifica con una concepción de lo propio ligada a la "privación" más que a la "apropiación" (Murillo, 1996). Por 
ejemplo, ver la televisión en compañía de un bebé se considera mayormente tiempo de ocio y no tiempo de cuidado.

Este posible sesgo a la hora de analizar el tiempo de ocio y de cuidado está ligado al hecho de tener únicamente en cuenta la dimensión material, cuantitativa, lineal y secuencial de las actividades cotidianas en vez de atender a las vivencias subjetivas de las mismas. Las encuestas y los análisis de corte más cuantitativo han servido para medir el volumen del trabajo no remunerado que queda fuera de los análisis económicos tradicionales (Legarreta, 2008; Esquivel, 2011), así como para dotar de datos a muchas de las reivindicaciones feministas (Bryson, 2008). No obstante, cabe advertir también que tiene sus limitaciones a la hora de abordar aspectos fundamentales para analizar y comprender algunas de las características intrínsecas del trabajo doméstico-familiar: la intensidad, la disponibilidad o el "tiempo de presencia" (estar presente sin necesariamente estar llevando a cabo una actividad, como por ejemplo, cuando una criatura está durmiendo) y las dimensiones morales (sacrificio, abnegación, satisfacción por el deber cumplido... ) o políticas (apropiación, capacidad de decisión...) del tiempo, por ejemplo (Legarreta, 2008, 2012).

Desde una mirada tal, el tiempo emerge en las experiencias de las mujeres como un factor que a menudo produce malestar (Moreno, 2007) y la "doble jornada" se categoriza como "doble presencia" (Balbo, 1994), concepto que surge para abarcar una mayor complejidad pues "la doble presencia no significa solamente una doble jornada, sino una mayor intensidad de carga de trabajo que es asumida día a día por las mujeres de manera sincrónica y cotidiana" (Torns et al., 2007: 39). A partir de estas aportaciones se plantea dar un giro más a estas propuestas y se acuña la noción "doble presencia-ausencia" para hacer referencia a situaciones que implican "estar y no estar", dar saltos de un ámbito a otro (Izquierdo, 1998) y tener que compaginar cotidianamente "dos lógicas contrapuestas": la lógica mercantil de la acumulación de capital y la lógica del cuidado y de la sostenibilidad de la vida (Carrasco, 2013; Pérez Orozco, 2014). Es más, algunas autoras apuntan a que estas situaciones conllevan una falta de disposición de tiempo para cualquier otra actividad que vaya más allá del desempeño de estas dos ocupaciones (Balbo, 1994), especialmente, la participación sociopolítica (Carrasco, 2001). De esta forma, se ha llegado a afirmar que el doméstico 
familiar "[e]s un tipo de trabajo que no puede ser medido cuantitativamente. Lo que produce el trabajo afectivo (...) es intangible, y no siempre es material, ya que (...) está ligado a los sentimientos, las emociones y las energías" (Gutiérrez-Rodríguez, 2013: 132).

Teniendo en cuenta todo ello, es remarcable la propuesta de Miriam Glucksmann (2005) que entiende el "trabajo" desde una visión relacional que va más allá de una mera definición, proponiendo un término inclusivo que reconozca las características históricas, empíricas y teóricas del trabajo, poniendo atención a su articulación e interconexión entre las distintas actividades y relaciones que lo construyen. Por ello, lejos de comprender el trabajo desde un análisis dualista y dicotómico, esta autora propone un esquema múltiple e interrelacionado. Según esta perspectiva, el trabajo está necesariamente unido a la temporalidad, no obstante, entiende que una concepción del trabajo relacional e interconectada abarca dimensiones temporales del trabajo extensas y multifacéticas que precisan una aproximación compleja, no limitada al tiempo lineal y estándar, como ocurre en el caso de las encuestas de usos del tiempo.

En este contexto, la reflexión en torno a la noción "triple presenciaausencia" surge con la finalidad de dar respuesta a dos necesidades: por un lado, la de incorporar la participación sociopolítica al análisis de la "doble presencia-ausencia" (Izquierdo, 1998) y, por otro, la de introducir la dimensión subjetiva al estudio de la "carga total de trabajo" (Moreno y Borràs, 2013).

\section{ApREndizaje DE GÉnERo y DISTRIBUCIÓN DEL TRABAJO DOMÉSTICO-FAMILIAR}

\subsection{Enfoques teóricos sobre la distribución del trabajo doméstico-familiar}

Siguiendo otros trabajos (Dominguez-Folgueras, 2015; Ajenjo y García, 2014; Carrasco y Domínguez, 2011; Sayer, 2005), se puede afirmar que el estudio de la distribución del trabajo doméstico y los cuidados se ha desarrollado principalmente a través de tres enfoques teóricos: disponibilidad de tiempo, recursos económicos y efecto de género. Si bien mayormente se han desarrollado de forma independiente, estos enfoques pueden considerarse complementarios. El enfoque de la 
disponibilidad de tiempo está ligado a las teorías sobre el capital humano y las teorías sobre la familia. Sostiene que los diferentes miembros del hogar contribuyen al desarrollo del trabajo domésticofamiliar de acuerdo a sus habilidades, a la productividad (como forma de maximizar la utilidad) y al tiempo que socialmente se espera que dediquen. La decisión es fruto de un cálculo de oportunidades racional. El de los recursos relativos entiende el reparto del trabajo domésticofamiliar como consecuencia de una negociación entre los miembros del hogar determinada por factores que definen las posibilidades de negociación (estatus social, nivel de estudios, de ingresos...). La perspectiva del efecto de género (doing gender) subraya los límites de los enfoques anteriores, puesto que no consideran las relaciones de poder basadas en el género, ni el efecto de las normas sociales. Sostiene que la forma en la que se organiza el ámbito doméstico-familiar se define y se expresa por las relaciones de género. Los individuos performan cotidianamente el género a través de su comportamiento. De esta forma, si los dos primeros enfoques ponen el foco de atención en los aspectos materiales como los recursos monetarios o el tiempo entendido como recurso abstracto y cuantificable, el tercero hace referencia a aspectos subjetivos como la socialización en base al género.

Diferentes trabajos han subrayado la fuerza explicativa de esta última teoría, ya que permite arrojar luz sobre dimensiones que las dos anteriores no han podido explicar (Sevilla-Sanz, Gimenez-Nadal y Fernández, 2010; Ajenjo y García, 2014). Desde esta mirada, se apunta a la existencia de normas de género cuyo efecto supone que, a partir de cierto punto, la tendencia hacia el reparto igualitario del trabajo doméstico y los cuidados en los hogares desaparece (Coltrane, 2000). Según esta perspectiva, el género se "hace" (doing gender) o reproduce a través de las relaciones interpersonales que reflejan ideologías y patrones culturales, por tanto, todas nuestras interacciones construyen (o deconstruyen) el género normativo y el ámbito doméstico-familiar es un espacio privilegiado para dar cuenta de las formas de "hacer género". De esta forma, las pautas de uso del tiempo nos otorgan indicios interesantes acerca de la forma de "hacer" o "deshacer" el género a través de las interacciones (Bryson, 2007). 


\section{2. "Haciendo" y "deshaciendo" el género}

La perspectiva del efecto de género (doing gender) ha sido propuesta por West y Zimmerman (1987) y desarrollada posteriormente, entre otras, por Coltrane (2000). Según West y Zimmerman (1987), las normas de género hegemónicas estipulan que las mujeres deben realizar la mayor parte del trabajo doméstico y de cuidado para reafirmar su feminidad, mientras que los hombres deberán realizar menos para mantener su masculinidad. En esta línea, Coltrane (2000) señala que mientras el trabajo doméstico se guía a través de las normas masculinas, el trabajo de cuidados se guía por las femeninas. Es decir, la presencia de los hombres en el desempeño del trabajo doméstico supone perder su "estatus" masculino, mientras que la ausencia de las mujeres en el cuidado supone una pérdida de su feminidad.

En este sentido, la investigación de Sevilla-Sanz, Gimenez-Nadal y Fernández (2010) apunta que un salario más alto no es siempre muestra de un mayor poder de negociación de las mujeres $y$, por ende, un reparto del trabajo no remunerado más igualitario; sino que cuando el nivel salarial de ellas comienza a superar al de sus parejas la tendencia al reparto igualitario se estanca. De este modo, señalan que las mujeres que han pasado a ser las principales "ganadoras de pan" realizan mayor volumen de trabajo doméstico y de cuidado que aquellas que ganan lo mismo que sus parejas, como forma de compensar el desvío de la norma de género. En esta línea, el trabajo de Cyrino (2012) sobre mujeres ejecutivas en Brasil muestra que, formando parte en su entorno laboral de un grupo muy masculinizado y con unas lógicas consideradas masculinas, las mujeres ejecutivas refuerzan su rol de género en el hogar para reafirmar su identidad femenina, no tanto a través del desempeño de las ocupaciones concretas (puesto que éstas son mayoritariamente externalizadas), sino haciendo suyo el control de la gestión del trabajo de las trabajadoras domésticas contratadas para llevarlas a cabo. En otros casos, sin embargo, como veremos más adelante, el desempleo para los hombres puede conllevar una mayor implicación en el ámbito doméstico-familiar y cierta tendencia a "deshacer" las normas de género hegemónicas.

A través del análisis de los datos de las Encuestas de Presupuestos de Tiempo de Eustat (1993-2013) se concluye que en la medida en que las mujeres han aumentado su presencia en el mercado laboral, ha 
disminuido su implicación en el trabajo doméstico, posiblemente debido a una merma en su disponibilidad de tiempo y al aumento de recursos relativos, sin embargo, no podemos decir lo mismo del cuidado, ya que ellas han mantenido la tendencia de responsabilizarse de este trabajo a lo largo de los veinte años estudiados. Por lo tanto, es posible afirmar que el espacio de cuidado se reafirma como espacio de la construcción de la feminidad (Sagastizabal y Luxán, 2015). En el caso de los hombres, a pesar de que su implicación sea considerablemente menor que la de las mujeres, se puede apreciar una tendencia hacia una mayor participación en el ámbito doméstico-familiar $y$, en consecuencia, un posible cuestionamiento del modelo de masculinidad tradicional. De este modo, si se observa su dedicación en el trabajo doméstico, sin tomar en consideración los cuidados (Legarreta y García Sainz, 2015), se constata una implicación mayor en 2013 que en 1993: un 20\% más de hombres dedica parte de su tiempo diario al trabajo doméstico, empleando para tal fin 15 minutos más. Así, si en 1993 seis de cada diez hombres participa en estas ocupaciones, en 2013 lo hace ocho de cada diez. El tiempo que emplean los hombres que efectivamente lo desempeñan pasa de 1 hora y 45 minutos en 1993 a 2 horas, veinte años más tarde. No obstante, su mayor implicación no es constante ni gradual, sino que se caracteriza por altibajos, mostrando un descenso de siete puntos porcentuales en los últimos cinco años estudiados.

\subsection{Avances hacia relaciones de género más igualitarias}

Se puede afirmar, por tanto, que la mayor participación de los hombres en el ámbito doméstico-familiar muestra una tendencia incipiente que, aunque se ha llegado a definir como "irrisoria", da muestras de posibilidades de cambio (Sullivan, 2000). De esta forma, sin olvidar la persistencia de las desigualdades, se ha apuntado una tendencia hacia un cambio del contrato social (Folbre y Nelson, 2000), llegando a afirmar que la "era del salario familiar" está en crisis (Fraser, 2000). Así, si bien algunos trabajos advierten de que la implicación de los hombres en el ámbito doméstico-familiar depende en mayor medida de las características de sus parejas que de las suyas propias (González y Jurado-Guerrero, 2009; Ajenjo y García, 2014; Agirre, 2015), otros apuntan que la crisis productiva-financiera puede dificultar para muchos el establecimiento de arreglos familiares tradicionales ("modelo hombre ganador de pan") y propiciar la construcción de relaciones de 
género más simétricas (Abril et al., 2015). En este sentido, se pone de manifiesto que la crisis del empleo y la proliferación de la flexibilidad y la precariedad laboral han dado paso a la emergencia de identidades que se estructuran cada vez en menor medida a través del empleo "tradicional". Así, los hombres con trayectorias laborales más inestables y caracterizadas por la precariedad, son los que muestran una mayor tendencia hacia el reparto igualitario del trabajo doméstico y de cuidado, ya que ello no les supone una pérdida de prestigio o estatus, debido a que se encuentran de por sí en una situación en la que carecen de ello (Abril et al., 2015; Torns et al., 2011).

Asimismo, estos cambios han abierto también la posibilidad de visualizar otros tiempos que van más allá de los guiados por la lógica mercantil (Leccardi, 1996), a pesar de que éstos continúan siendo los hegemónicos. Estas otras formas de comprender y experimentar el tiempo son propias de las relaciones sociales, del cuidado, son tiempos que poco tienen que ver con la lógica de la eficiencia y la rapidez de la productividad (Adam, 2003), sino que están ligados a los ritmos de los procesos naturales, biológicos y sociales. Entendido de este modo, el tiempo puede constituir una experiencia positiva, de tiempo propio o tiempo "para sí" o, por el contrario, puede ser vivido como fuente de malestar, como un tiempo "impuesto" sobre el cual no hay poder de decisión, como ocurre en algunos casos con el tiempo dedicado a los y las demás, en relación tanto al tiempo de cuidados como al dedicado al trabajo voluntario realizado en el ámbito de la participación sociopolítica (Sagastizabal, 2014). Arneil (2006) señala que, a menudo, cuando los hombres se involucran en el ámbito participativo crean capital social para sí, para ellos mismos (a través de redes de apoyo, económicas, de prestigio...), mientras que las mujeres suelen crear capital social para los demás. Además, debido a la influencia de la ideología de género, muchas actividades realizadas por las mujeres en este ámbito no se consideran políticas, ni por los estudios de corte androcéntrico, que sólo entienden como participación política aquella llevada a cabo en ámbitos formales e institucionales, ni por las propias mujeres. Así, algunas investigaciones han puesto el foco de atención en este tipo de participación "invisible" (Hernández, 2008; Coffé y Bolzendahl, 2010; Hooghe y Stolle, 2004) o invisibilizada. Incluso, otras autoras han subrayado el carácter político del trabajo de cuidado (Herd y Harrington, 2002). Por consiguiente, se puede afirmar que tanto las 
formas de participar en el ámbito sociopolítico como las maneras de visibilizar dicha participación pueden llegar a "hacer" o "deshacer" el género.

\section{MÁs ALLÁ de LA MATERIALIDAD: LA ÉTICA DEL CUIDADO y LOS ASPECTOS SUBJETIVOS}

\subsection{Fundamentos de la ética del cuidado}

La perspectiva en torno a la ética del cuidado se desarrolla a través de la obra de Carol Gilligan In a diferente voice (1982) a partir de la crítica al trabajo de Lawrence Kohlberg en relación a la psicología del desarrollo moral. Gilligan argumenta que, a diferencia de los razonamientos de los hombres - formales y abstractos-, las experiencias morales de las mujeres, desarrolladas a través del cuidado, se basan en razonamientos contextuales y narrativos. De esta forma, señala la diferencia entre la ética de la justicia y la ética del cuidado. La ética del cuidado se fundamenta en el desarrollo de disposiciones morales y no tanto en el aprendizaje de principios (ética de la justicia), y de este modo, privilegia unas respuestas contextuales y específicas así como el punto de vista del "otro concreto" (Benhabib, 1990). La obra de Gilligan sitúa el cuidado en el debate sobre la justicia y la ética, sacándolo de los hogares, para instalarlo en la esfera pública (Vega, 2009).

Tal como apunta Benhabib (1990), el debate en torno a la ética del cuidado y la ética de la justicia tiene su origen en la crítica a las teorías morales universalistas presentes principalmente en las corrientes de pensamiento tradicional-liberal occidental desarrolladas en torno a la teoría del contrato social. Estas teorías, apunta la autora, son sustitucionalistas, en el sentido de que el universalismo que defienden identifica las experiencias de un grupo específico de sujetos (los hombres) como caso paradigmático de la humanidad. Frente a esta mirada, que invisibiliza las experiencias de las mujeres, se reivindica su validez e importancia tanto moral como política, de modo que las cuestiones sobre la moralidad y la ética entran en la agenda política feminista (Sevenhuijsen, 1998).

Desde una mirada feminista, la ética del cuidado y la ética de la justicia no se presentan de forma contrapuesta, sino complementaria. Así, las teóricas sobre la ética del cuidado adoptan también una perspectiva 
sobre la justicia. Desde esta mirada se defiende que la perspectiva de la ética del cuidado requiere pensar la justicia "en función de las circunstancias y de los casos concretos, y no simplemente como un conjunto general de principios cuya aplicación es dejada al juicio de los tribunales, de los políticos o de los filósofos" (Tronto, 2008: 18, citado en Paperman, 2011: 29).

\subsection{Nuevas aperturas}

Partiendo de los trabajos de Gilligan, se apela a una dimensión de la justicia que se desarrolla en el plano cotidiano y contextual y que incluye un sentido de la vulnerabilidad, así como de las exigencias morales y políticas que dicha vulnerabilidad implica (Paperman, 2011). De este modo, se cuestiona la imagen del individuo autónomo, como aquel que nace y crece sin necesidad de los demás - representada en la figura del hongo hobbesiano a la que hace referencia Benhabib (1990)-, al tiempo que se favorece una noción de vulnerabilidad intrínseca al ser humano en tanto que "homo vulnerabilis" (Molinier, 2011). La ética del cuidado reconoce de manera más realista de lo que lo hacen las teorías sociales y morales "mayoritarias" que la dependencia y la vulnerabilidad no son accidentes del camino, que solo les pasan a los demás (a los "otros"), sino que son rasgos inherentes a la condición humana (Paperman, 2004). Así, la vulnerabilidad se define como un atributo que pasa a formar parte del patrimonio de lo común (L. Gil, 2011): cada uno/a podemos reconocernos en el otro/a a través de nuestra propia condición inestable, precaria en parte, contingente, circunscrita al entorno que nos rodea, e interdependiente.

Al desvanecerse la idea de la autonomía plena, se rompe con la diferenciación entre una parte de la población que precisa cuidados y otra que los presta, de modo que se pone de manifiesto el carácter imprescindible del trabajo doméstico y los cuidados para la subsistencia humana (Carrasco, 2001). Desde esta perspectiva, se hace necesario conjugar la dimensión sincrónica, cotidiana, del tiempo de cuidados con su carácter diacrónico: todas las personas precisan cuidados a lo largo de toda la vida y no solamente en determinados momentos (aunque se intensifiquen en situaciones de enfermedad y al inicio y final de la vida), por lo que las relaciones sociales establecidas en torno a la dependencia y a la vulnerabilidad se despliegan a través de un tiempo prolongado, a 
menudo el tiempo de toda una vida (Damamme, 2011; Damamme y Paperman, 2009).

Asimismo, la perspectiva de la ética del cuidado saca a la luz los aspectos subjetivos y emocionales, al definir el cuidado no sólo como "una actividad con un contenido concreto, sino una disposición ética basada en la responsabilidad y la atención a las necesidades y juicios de los demás que puede atravesar distintas actividades, espacios, sujetos y relaciones" (Vega, 2009: 83). De este modo, puede trasladarse también al ámbito de la participación sociopolítica, subrayando la pertinencia de tener en cuenta el contexto y las experiencias subjetivas (Taylor, 2005), así como las emociones y sentimientos presentes en este ámbito como el sentido de pertenencia, de responsabilidad, de compromiso y de obligación moral (Sagastizabal, 2014; Goss, 2003), relacionados con una experiencia de la participación no tanto para sí, sino para los demás (Arneil, 2006). Prestar atención a la dimensión subjetiva no conlleva, sin embargo, olvidar que el cuidado "es ante todo trabajo" (Molinier, 2011) y se hace el esfuerzo por subrayar la pertinencia de abordar su materialidad, pues se entiende que "el concepto de care permite no disociar trabajo y ética" (Paperman, 2011). De esta forma, en tanto que disposición, el tiempo de cuidado se vuelve más cercano a la dimensión temporal que descansa sobre la noción griega kairos (el tiempo de la sazón, del momento oportuno) (Legarreta, 2009), mientras que, en cuanto trabajo, puede ser medido y cuantificado a través de una concepción del tiempo abstracta y descontextualizada, cronos.

\subsection{Las críticas y sus respuestas}

Pese a su potencialidad, cabe señalar que la ética del cuidado ha sido criticada por su esencialismo, advirtiendo que tanto desde una perspectiva histórica (a lo largo del tiempo), antropológica (en relación a otras culturas) como sociológica (desde una mirada de clase) se puede apreciar que los atributos que se asocian con el cuidado se definen en relación a una forma específica de entender la maternidad (Torns, 2007) que toma como referencia a la mujer desde una perspectiva biológica, considerándola, en tanto que sujeto político, como grupo homogéneo (Artiaga, 2009). Tales críticas son asumidas también por autoras que se posicionan en la perspectiva de la ética del cuidado pero que se desvinculan de dicha forma específica de entenderla, pues la asocian principalmente a una corriente "maternalista", ejemplificada entre 
otros, en el trabajo de Virginia Held, Sandra Ruddick y Nel Noddings (Martín-Palomo, 2010), que define la ética del cuidado como "ética femenina" y no como "ética feminista" (Paperman, 2011).

Frente a las posturas esencialistas y los argumentos biologicistas, se defiende, siguiendo a Joan Tronto (1987), que el cuidado no es una disposición moral específica de las mujeres sino un efecto de su posición social subalterna. Tronto argumenta que la ética de la voz diferente no es exclusiva de las mujeres, sino de aquellas personas, principalmente mujeres, cuya experiencia vital está basada en el trabajo específico de ocuparse de los y las demás (Tronto, 1987). Como apuntan Arango y Molinier (2011), esta apreciación resulta importante, pues permite desnaturalizar la ética del cuidado en un sentido doble: por un lado, situando su emergencia en una "actividad", el trabajo doméstico y los cuidados, y no en una pretendida "naturaleza" biológica de las mujeres y, por otro, estableciendo diferencias entre las mujeres, pues no todas están concernidas de la misma forma por las actividades de cuidado.

En esta línea, se advierte también del peligro de esencializar o mistificar el cuidado, identificándolo como "uno y auténtico" (Vega, 2009). Así, en el plano analítico se aboga por una noción de cuidado multidimensional, compleja, contextual y que encierra tensiones, como "un conjunto de prácticas cambiantes vinculadas a cualidades, valores, regulaciones igualmente cambiantes" (Vega, 2009: 88). El cuidado se define a menudo como aquello que se hace con amor y/o por amor y en relación a las personas del entorno más cercano. No obstante, no siempre cumple con los requisitos de amor (Torns, 2007), a veces, es causa y objeto de relaciones perversas de dominación, está lejos de ser siempre gratificante (L. Gil, 2011), y puede derivar en una relación de sumisión, de abuso de poder o de abandono (Martín-Palomo, 2010).

Las relaciones de cuidado son complejas y caracterizadas por la ambivalencia, pues a menudo los afectos y las emociones, el amor y la gratificación, se entremezclan con sentimientos de sacrificio, culpa, privación, obligatoriedad, abusos, chantajes emocionales... (BianchiPernasilici, 2014). Asimismo, el poder se puede ejercer de forma desmedida tanto por parte de la persona que cuida hacia la persona que es cuidada, como a la inversa, pues las relaciones de dominación no siempre toman la misma dirección, sino que en su configuración entran en juego diferentes aspectos (a menudo interrelacionados entre sí) 
determinados por la posición de cada persona implicada en la relación: género adscrito, posición social, etnia, lugar de procedencia, edad, estatus legal, posición dentro de la familia... Del mismo modo, la proximidad tampoco es constitutiva de las relaciones y prácticas de cuidado: hay diferentes maneras de hacerse cercano/a o, al contrario, de alejarse de una situación cuyos "detalles" son importantes, de responder o no, de ver o de ignorar las exigencias (Paperman, 2011). Además, las prácticas y representaciones del cuidado se definen en relación a un sistema normativizado que determina la distinción entre el buen cuidado y el mal cuidado. En otros trabajos se pone de manifiesto que la frontera entre ambos es difícil de determinar sin tomar en consideración las situaciones específicas y contextualizadas propias del cuidado y se pone el acento en las relaciones de respeto y de reconocimiento mutuo entre las personas involucradas en la relación y no sólo en las prácticas llevadas a cabo (Molinier, 2011).

De esta forma, cabe diferenciar entre la experiencia cotidiana del cuidado (plano empírico) y la dimensión política de la ética del cuidado. Como subraya la propia Carol Gilligan, en el plano empírico la ética del cuidado es una ética femenina porque se desarrolla en el marco de una estructura binaria y jerárquica, la del patriarcado. Sin embargo, en una sociedad no configurada sobre las desigualdades de género, la ética del cuidado es una ética universal sobre la que se asiente en un modelo de ciudadanía inclusiva:

"En un contexto patriarcal, el cuidado es una ética femenina. Cuidar es lo que hacen las mujeres buenas, y las personas que cuidan realizan una labor femenina; están consagradas al prójimo, pendientes de sus deseos y necesidades, atentas a sus preocupaciones; son abnegadas. En un contexto democrático, el cuidado es una ética humana. Cuidar es lo que hacen los seres humanos; cuidar de uno mismo y de los demás es una capacidad humana natural. La diferencia no estaba entre el cuidado y la justicia, entre las mujeres y los hombres, sino entre la democracia y el patriarcado. (...) Las diferencias de género en la voz moral no son producto de la naturaleza o de la crianza en sí, sino del modelo binario y jerárquico fundamental para el establecimiento y la conservación de un orden patriarcal" (Gilligan 2013: 50).

A nivel propositivo y político, por tanto, "la ética del cuidado no es una ética femenina, sino feminista, y el feminismo guiado por una ética del 
cuidado podría considerarse el movimiento de liberación más radical en el sentido de que llega a la raíz- de la historia de la humanidad" (Gilligan, 2013: 31).

\section{LA "TRIPLE PRESENCIA-AUSENCIA": UNA PROPUESTA EN CONSTRUCCIÓN ${ }^{7}$}

\subsection{Definiendo la "triple presencia-ausencia"}

Tal como señala Paperman (2011), la perspectiva del cuidado requiere del desarrollo de nuevas herramientas que se adopten al conocimiento de dichas actividades. Según esta autora, deben ser herramientas que permitan su descripción como trabajo, utilizando un concepto amplio que incluya la dimensión moral (subjetiva, emocional y relacional, cabría añadir) de las actividades y que, asimismo, ofrezca la posibilidad de ver y pensar realidades que han sido ignoradas o mal comprendidas. Además, la perspectiva del cuidado posee capacidades descriptivas para explicar cómo se sostiene la sociedad apoyándose en un trabajo que no ha sido suficientemente reconocido. Centrándose en la noción de trabajo, Glucksmann (2005) apunta la necesidad de reexaminar la naturaleza del concepto, para evitar el encaje de nuevas y complejas prácticas en viejas categorías dicotómicas (trabajo vs. no trabajo, público vs. privado), creando marcos conceptuales con mayor número de matices, así como herramientas analíticas inclusivas que tengan en cuenta las actividades que tradicionalmente han quedado en los márgenes de la forma convencional de entender el "trabajo", como el cuidado o la participación sociopolítica.

El estudio de la "triple presencia-ausencia" pretende ofrecer la posibilidad de dar un paso en este camino. Con tal finalidad, parte de una definición o comprensión amplia del trabajo, en la que se enmarcan tanto el asalariado como el doméstico-familiar y el voluntario realizado

\footnotetext{
${ }^{7}$ Cabe advertir que en estos momentos la "triple presencia-ausencia" es una propuesta que está en proceso de construcción y que ha sido desarrollada principalmente en su dimensión analítica. Hoy por hoy, ésta es sin duda una de sus limitaciones. Una primera validación en el plano empírico ha sido realizada en la investigación llevada a cabo por Marina Sagastizabal como Trabajo Fin de Máster en el marco del Máster de Estudios Feministas y de Género de la UPV/EHU. En estos momentos la propuesta está siendo desarrollada con mayor profundidad a través de la investigación que está llevando a cabo Marina Sagastizabal en su tesis doctoral inscrita en el Departamento de Sociología 2 de la UPV/EHU bajo la dirección de las Profesoras Teresa Torns y Matxalen Legarreta.
} 
en el ámbito de la participación sociopolítica. De este modo, se relacionan los cuidados no sólo con la esfera productiva-mercantil como se propone en planteamientos predecesores en torno a la "doble jornada" (Hochschild y Machung, 2003; Durán, 1986) o la "doble presencia-ausencia" (Izquierdo, 1998)-, sino también con la participación sociopolítica, para visibilizar la relevancia social y analítica de esta última. Así, se considera que la participación sociopolítica es, además de un trabajo, un pilar fundamental en nuestra sociedad, pues se entiende en su dimensión más cotidiana, informal y, a menudo, invisibilizada: a saber, como aquella que no se ciñe a lo estrictamente público e institucional. Por tanto, se pone el foco de atención en su plano "invisible", en el que, principalmente, son protagonistas las mujeres: actividades que a menudo no se realizan en la esfera pública, pero que la sostienen y la posibilitan. Se pretende, de esta forma, dar voz a otras formas de hacer política, tradicionalmente protagonizadas por mujeres, para identificar y sacar a la luz las prácticas y experiencias desarrolladas desde una óptica que tiene en cuenta la ética del cuidado y que ponen, o se esfuerzan por poner, la sostenibilidad de la vida en el centro, cuestionando la figura del "militante champiñón" (Sagastizabal, 2014), aquella persona que se dedica "al cien por cien" a la participación sociopolítica gracias al trabajo invisible de otras personas, que cubren en el día a día sus necesidades materiales y afectivas.

\subsection{Potencialidad de la propuesta}

En el plano analítico, el estudio sobre la "triple presencia-ausencia" se nutre de los debates académicos desarrollados en el campo de la teoría feminista sobre las concepciones de ciudadanía, trabajo y tiempo, poniendo de manifiesto el sesgo de género presente en las mismas y proponiendo otras definiciones que enriquezcan el análisis e incluyan experiencias que no se limiten a la esfera pública y masculina hegemónica. Para ello, se apuesta por analizar el ámbito cotidiano de la vida de las personas, donde lo público y lo privado se entrelazan y donde el tiempo se convierte en una categoría analítica privilegiada. La finalidad es dar cuenta de las relaciones sociales, de los ritmos y las estrategias que se desarrollan para llevar a cabo dichas actividades de forma sincrónica y cotidiana, así como a lo largo de toda una vida, en los diferentes momentos del ciclo vital, para estudiar los obstáculos, las desigualdades y el malestar que genera la organización social actual, 
que dificulta, cuando no imposibilita, la conciliación entre esferas, espacios, tiempos y funciones, abocando a muchas personas a "permanentes malos arreglos" (Torns, 2005) e incluso, obligando a muchas de ellas a abandonar el terreno sociopolítico (principalmente mujeres que desempeñan labores de cuidado de forma intensiva).

¿Quién se ocupa y se preocupa de qué y cómo?, ¿cuándo?, ¿dónde?, ¿con qué intensidad? A través del ejercicio de dar respuesta a estas preguntas puede ser descrita, evaluada y revisada la "organización social y política del cuidado", en el sentido que le otorgan al término Mary Daly y Jean Lewis (2000) en su propuesta en torno al social care, tomando en cuenta no sólo las actividades y sus significados, sino también "la normatividad, los costes y los contextos sociales dentro de los cuales este trabajo es asignado y llevado a cabo" (Lewis, 1998:6) o no. Se pretende contestar a dichas cuestiones a través del análisis de la "triple presencia-ausencia", teniendo en cuenta cómo las actividades son coordinadas en el interior de un contexto que las invisibiliza y las devalúa, o las idealiza a través de un discurso de la domesticidad (Legarreta, 2012). Asimismo, mediante esta propuesta, se pretenden analizar las trayectorias de las personas que "están y no están" al mismo tiempo en estos ámbitos, poniendo el foco de atención en la sincronía y el "encaje" temporal (Lewis y Weigther, 1992).

En este sentido, se considera que el tiempo es también una categoría que debe ser cuestionada. Este cuestionamiento conlleva una comprensión del mismo que no se limita a la mera suma lineal de horas, minutos y segundos, sino que pone atención en otras dimensiones, cíclicas, biológicas y sociales, ligadas al contexto y las relaciones, pues “los ritmos del cuerpo son inseparables de las personas, el bienestar y de los ritmos cotidianos de la vida" (Adam, 1995:45). Así, desde una mirada feminista, se aboga por una noción de tiempo, plural, no-jerárquica, multidimensional y unida a la experiencia (Leccardi, 1996; Adam, 1989; Legarreta, 2012). Por tanto, el tiempo constituye una categoría analítica clave en el estudio de la "triple presencia-ausencia", tanto en su dimensión material como subjetiva. De este modo, como se ha apuntado anteriormente, se incorpora la dimensión subjetiva al análisis de la "carga total del trabajo" propuesta por Moreno y Borrás (2013). Con todo, a través de esta propuesta se pretende ahondar en las experiencias y vivencias de las personas que experimentan una "triple 
presencia-ausencia", apoyándose en los puntos de vista y en las experiencias de los sujetos que la protagonizan. En base a las presencias-ausencias en estos tres ámbitos, es posible comprender qué "funciona o deja de funcionar" (Paperman, 2011) tanto a nivel social como en el ámbito de los hogares a la hora de garantizar de forma satisfactoria la provisión de cuidados.

Asimismo, a nivel analítico la propuesta en torno a la "triple presenciaausencia" pretende desligar el cuidado de la domesticidad y de las formas simbólicas de feminidad, atendiendo a las voces tanto de mujeres como de hombres implicados en el mismo a través de los significados otorgados al tiempo, así como a lo que supone "estar y no estar" en cada ámbito para cada persona. Con ello, se pretende también detectar las posibilidades de cambio en el plano simbólico y de avance hacia un reparto más equitativo y justo, dando cuenta de las dinámicas que se desarrollan en relación a las formas de "hacer y deshacer" el género.

Cabe señalar que la propuesta en torno a la "triple presencia-ausencia" nace con la pretensión de desarrollarse desde una dimensión analítica, empírica y política. Así, encaja dentro del debate más amplio sobre la definición de la ciudadanía desde la perspectiva de la ética del cuidado, preguntando en qué contexto político, social y económico se puede desarrollar una ética feminista del cuidado, otorgando, además, una dimensión práctica al debate: ¿cómo nos organizamos para conseguirlo?

A lo largo del texto hemos presentado la propuesta atendiendo únicamente a su plano analítico y poniendo de manifiesto su potencialidad. Queda, para futuros trabajos, contrastarla a nivel empírico y sacar a la luz sus posibles limitaciones.

\section{Bibliografía}

Abril, P., Amigot, P., Botía-Morillas, C., Dominguez-Folgueras, M. González, M. J., 2015, “Ideales igualitarios y planes tradicionales: análisis de parejas primerizas en España", en Reis, 150 (Abril-Junio), pp. 3-22.

Adam, B., 1989, "Feminist social theory needs time. Reflections on the relation between feminist thought, social theory and time as an 
importan parameter in social análisis", en Sociological Review, 37 (3), pp.458-473.

Adam, B., 1995, Timewach. The social analysis of time, Polity Press, Cambridge.

Adam, B., 2003, "When time is money: contested rationalities of time in theory and practice of work", en Theoria: A Journal of Social and Political Theory, 102, pp. 94-125.

Agirre, A., 2015, Negoziazio prozesuak bikote eredu berdinzaleetan, Tesis doctoral inédita, Universidad del País Vasco, Leioa.

Ajenjo, M., García, J., 2014, “Cambios en el uso del tiempo de las parejas ¿estamos en el camino hacia una mayor igualdad", en Revista Internacional de Sociología, 72 (2), pp. 453-476.

Alcañiz, M., 2015, “Género con clase: la conciliación desigual de la vida laboral y familiar", en RES, 23, pp. 29-55.

Alonso, L. E., 2007, La crisis de la ciudadanía laboral. Autores, textos y temas, Vol. 55, Anthropos, Barcelona.

Anderson, B., 2000, Doing the dirty work? The global politics of domestic labour, Zed Books, London.

Arango, L. G., Molinier, P., 2011, “El cuidado como ética y como trabajo", en L. G. Arango y P. Molinier (Comps.), El trabajo y la ética del cuidado, Universidad Nacional de Colombia, La Carreta, Medellín, pp. 15-24.

Arneil, B., 2006, "Just communities. Social capital, gender, and culture", en B. O'Neill y E. Gidengil (Eds.), Gender and social capital, Routledge, London, pp.15-43.

Artiaga, A., 2009, “La producción política de la categoría de cuidados: voces de los movimientos feministas y marcos interpretativos de la ley de dependencia", en I Congreso Anual de la Red-ESPANET: Treinta años de Estado de bienestar en España, logros y retos para el futuro, 5-7 de Noviembre, Oviedo.

Balbo, L., 1994, "La doble presencia", en C. Borderías, C. Carrasco y C. Almenany (Comps.), Las mujeres y el trabajo. Rupturas conceptuales, Icaria, Barcelona, pp. 505-513.

Benhabib, S., 1990, “El Otro generalizado y el Otro concreto: la controversia Kohlberg-Gilligan y la teoría feminista", en S. Benhabib y D. Cornell (Eds.), Teoría feminista y teoría crítica, Edicions Alfons el Magnanim,Valencia, pp.119-149. 
Bianchi-Pernasilici, G. M., 2014, Migraciones y trabajo doméstico-de cuidados. el caso de la población andina en el gran Bilbao, Tesis doctoral inédita, Universidad del País Vasco, Leioa.

Borderías, C., Carrasco, C., Alemany, C., 1994, Las mujeres y el trabajo. rupturas conceptuales, Icaria, Barcelona.

Bryson, V., 2007, Gender and the politics of time: feminist theory and contemporary debates, Policy Press, Bristol.

Bryson, V., 2008, "Time-use studies a potentially feminist tool", en International Feminist Journal of Politics, 10 (2), pp. 135-153.

Carrasco, C., 2001. "La sostenibilidad de la vida humana: ¿Un asunto de mujeres?", en Mientras tanto, 82, pp. 43-69.

Carrasco, C., 2013, "El cuidado como eje vertebrador de una nueva economia", en Cuadernos de Relaciones Laborales, 31 (1), pp. 39-56.

Carrasco, C., Domínguez, M., 2011, "Family strategies for meeting care and domestic work needs: evidence from Spain", en Feminist Economics, 14 (4), pp. 159-188.

Carrasquer, P., 2002, “ ¿En los límites de la modernidad? Trabajo y empleo femenino precario en España", en Sistema, 167, pp. 73-94.

Carrasquer, C., Torns, T., Tejero, E., Romero, A., 1998, “El Trabajo Reproductivo", en Papers, 55, pp. 95-114.

Coffé, H., Bolzendahl, C., 2010, "Same game, different rules? Gender differences in political participation", en Sex Roles, 69, pp. 318-333.

Coltrane, S., 2000, "Research on household labor: modeling and measuring the social embeddedness of routine family work", en Journal of Marriage and the Family, 62 (Noviembre), pp. 1208-1233.

Cordoni, E., 1993, "Las mujeres cambian los tiempos", en Cuadernos de Relaciones Laborales, 2, pp. 281-299.

Cyrino, R., 2012, Mulheres executivas divisão do trabalho doméstrico a luz dos estereótipos de gênero, Belo Horizonte, MG Brasil.

Daly, M., Lewis, J., 2000, "The concept of social care and the analysis of contemporary welfare status", en British Journal of Sociology, 51 (2), pp. 281-298.

Damamme, A., 2011, "El care en las familias. Perspectiva temporal versus radiografía", en L. G. Arango y P. Molinier (Comps.), El Trabajo y La Ética Del Cuidado, Universidad Nacional de Colombia, La Carreta, Medellín, pp. 157-168. 
Damamme, A., Paperman, P., 2009, "Care domestique: de histories sans début, sans milieu et sans Fin", en Multitudes, 37, pp. 98-105.

Dominguez-Folgueras, M., 2015, “Parentalidad y división del trabajo doméstico en España, 2002-2010", en Reis, 149 (Enero-Marzo), pp. 45-64.

Durán, M. A., 1986, La jornada interminable, Icaria, Barcelona.

Esquivel, V., 2011, “Sixteen years after Beijing: What are the new policy agendas for time-use data collection", en Feminist Economics, 17 (4) (10/01), pp. 215-238.

Folbre, N., Nelson, J. A,. 2000, "For love or money-or both", en Journal of Economic Perspectives, 14 (4), pp. 123-140.

Fraser, N., 2000, "After the family wage: a postindustrial thought experiment", en B. Hobson (Ed.), Gender and Citizenship in Transition, Routledge, New York, pp. 1-32.

Galcerán, M., 2006, "Introducción: producción y reproducción en Marx", en Laboratorio Feminista (Ed.), Transformaciones del trabajo desde una perspectiva feminista: producción, reproducción, deseo y consumo, Tierra de Nadie, Madrid, pp. 13-26.

García Sainz, C., 2002, "Entre el valor y precio. Notas sobre una valoración económica del trabajo no remunerado", en VV.AA., Agor@ 2001: jornades per la integració de l'economia domèstica en el sistema econòmic global. Treball real, economia invisible, Generalitat de Catalunya, Institut Català de la Dona, Barcelona.

Gershuny, J., Robinson, J. P., 1988, “Historical changes in the household division of labor", en Demography, (Pre-2011) 25 (4), pp. 537-552.

Gilligan, C., 2013, “El daño moral y la ética del cuidado", en C. Gilligan, La ética del cuidado, Cuadernos de la Fundació Víctor Grifols i Lucas Cap. 30, Fundació Víctor Grífols i Lucas, Barcelona, pp. 10-39.

Glucksmann, M., 2005, "Shifting boundaries and interconnections: extending the 'total social organisation of labour'", en The Sociological Review, 53, pp. 19-36.

González, M. J., Jurado-Guerrero, T., 2009 “¿Cuándo se implican los hombres en las tareas domésticas? Un análisis de la encuesta de empleo del tiempo", en Panorama Social (segundo semestre), pp. 65-81.

Goss, K., A, 2003, "Rethinking the political participation paradigm", en Women $\in$ Politics, 25(4), pp. 83-118. 
Gutiérrez-Rodríguez, E., 2013, “Trabajo Doméstico-Trabajo Afectivo: sobre la heteronormatividad y la colonialidad del trabajo en el contexto de las políticas migratorias de la UE", en Revista De Estudios Sociales, Enero-Abril (45), pp.123-134.

Herd, P., Harrington, M., 2002, "Carework: invisible civic engagement", en Gender and Society, 16 (5), pp. 665-688.

Hernández, J. M., 2008, Hacia una cartografía de la participación invisible. Proyectando mapas para la intervención local de las mujeres, Diputación de Barcelona, Barcelona.

Hochschild, A, R., Machung, A., 2003 [1989], The second shift: working families and the revolution at home, Penguin Books, London.

Hooghe, M., Stolle, D., 2004, "Good girls go to the polling booth, bad boys go everywhere", en Women $\in$ Politics, 26 (3), pp. 1-23.

Hughes, C., 2002, Key concepts in feminist theory and research, Sage Publications, London.

Izquierdo, M. J., 1998, El malestar en la desigualdad, Edición Cátedra, Col. Feminismos, Vol. 48, Madrid.

L. Gil, S., 2011, Nuevos feminismos. Sentidos comunes en la dispersión. Una historia de trayectorias y rupturas en el Estado español, Traficantes de Sueños, Madrid.

Leccardi, C., 1996, "Rethinking social time: feminist perspectives" en Time and Society, 5 (2), pp. 169-186.

Legarreta, M., 2008, "El tiempo donado en el ámbito doméstico. Reflexiones para el análisis del trabajo doméstico y los cuidados", en Cuadernos de Relaciones Laborales, 26 (2), pp. 45-69.

Legarreta, M., 2009, "Le temps donné dans le travail domestique et de care", en Multitudes, (37-38), pp. 106-112.

Legarreta, M., 2012, El tiempo donado en el ámbito doméstico-familiar. Estudio sobre el trabajo doméstico y de cuidados, Tesis doctoral inédita, Universidad del País Vasco, Leioa.

Legarreta, M., García Diez, S., 2015, "El contexto internacional: la CAE y su entorno", en M. Legarreta (Coord.), Dos décadas de cambio social en la C. A. de Euskadi a través del uso del tiempo. Encuesta de presupuestos de tiempo, 1993-2013, Monográfico, Instituto Vasco de Estadística-Eustat, Vitoria-Gasteiz, pp. 463-497.

Legarreta, M., García Sainz, C., 2015, "Las familias y el reparto del trabajo doméstico", en M. Legarreta (Coord.), Dos décadas de cambio social 
en la C. A. de Eushadi a través del uso del tiempo. Encuesta de presupuestos de tiempo, 1993-2013, Monográfico, Instituto Vasco de Estadística-Eustat, Vitoria-Gasteiz, pp. 109-147.

Letablier, M. T., 2007, “El trabajo de cuidados y su conceptualización en Europa", en C. Prieto (Ed.), Trabajo, género y tiempo social, Complutense y Hacer, Madrid, pp. 64-84.

Lewis, J., 1998. "Gender, social care and welfare regimes", en Journal of European Social Policy, 2 (3), pp. 159-173.

Lewis, J. D., Weigther, A., 1992, "Estructura y significado del tiempo social", en R. Ramos (Ed.), Tiempo y sociedad, Centro de Investigaciones Sociológicas, Madrid, pp. 89-132.

Martín-Palomo, M. T., 2010, "Autonomía, dependencia y vulnerabilidad en la construcción de la ciudadanía", en Zerbitzutan, 48, pp. 57-69.

Moghadam, V. M., 2003, "Engendering citizenship, feminizing civil society: the case of the Middle East and North Africa", en Women $G$ Politics, 25 (1-2), pp. 63-87.

Molinier, P., 2011, "Antes que todo, el cuidado es un trabajo", en L. G. Arango y P. Molinier (Comp.), El trabajo y la ética del cuidado, Universidad Nacional de Colombia, La Carreta, Medellín, pp. 45-64.

Moreno, S., 2007, Temps, treball i benestar: una aproximació des de la vida cuotidiana, Tesis Doctoral Inédita, Universitat Autònoma de Barcelona, Centre d'Estudis Sociològics sobre la Vida Quotidiana i el Treball del Departament de Sociologia, Barcelona.

Moreno, S., Borràs, V., 2013, El temps de treball remunerat i no remunerat. Ampliació de resultats de l'enquesta de l'us del temps 2010-2011, Universitat Autònoma de Barcelona, Centre d'Estudis Sociològics sobre la Vida Quotidiana i el Treball, Barcelona.

Murillo, S., 1996, El mito de la vida privada. De la entrega al tiempo propio, Siglo XXI, Madrid.

Paperman, P., 2004, "Perspectives féministes sur la justice", en L'Annèe Sociologique, 54 (2), pp. 413-434.

Paperman, P., 2011, "La perspectiva del care: de la ética a lo político", en L. G. Arango y P. Molinier (Comps.), El trabajo y la ética del cuidado, Universidad Nacional de Colombia, La Carreta, Medellín, pp. 25-44.

Pateman, C., 1995 [1988], El contrato sexual, Anthropos, Barcelona. 
Pateman, C., 1996, "Críticas feministas a la dicotomía público/privado", en C. Castells (Ed.), Perspectivas feministas en teoría política, Paidós, Barcelona, pp. 31-52.

Pérez Orozco, A., 2014, Subversión feminista de la economía. Aportes para un debate sobre el conflicto capital-vida, Traficantes de Sueños, Madrid.

Prieto, C., 2007, "Del estudio del empleo como norma social al de la sociedad como orden social", en Papeles del CEIC, 1, pp. 1-28.

Sagastizabal, M., 2014, "Vivencias del tiempo social: compaginar la participación política, el cuidado y el empleo", en E. Araújo, E. Duque, M. Franch y J. Durán (Eds.), Tempos sociais e o mundo contemporâneo. As crises, as fases e as ruturas, Centro de Estudos de Comunicação e Sociedade (CECS), Universidade do Minho, Braga, pp. 220-231.

Sagastizabal, M., Luxán, M., 2015, "Género Y Tiempo", en M. Legarreta (Coord.), Dos décadas de cambio social en la C.A de Eushadi a través del uso del tiempo. Encuesta de presupuestos de tiempo, 1993-2013, Monográfico, Instituto Vasco de Estadística-Eustat, VitoriaGasteiz, pp. 385-425.

Sayer, L. C., 2005, "Gender, time and inequality: trends in women's and men's paid work, unpaid work and free time", en Social Forces, 84 (1), pp. 285-303.

Scott, J. W., 1982, "The mechanization of women's work", en Scientific American, 247: 3, pp. 166-187.

Scott, J. W., 1993, "La mujer trabajadora en el siglo XIX", en G. Duby y M. Perrot (Dirs.), Historia de las mujeres, Tomo IV, Taurus, Madrid. pp. 427-461.

Sevenhuijsen, S., 1998, Citizenship and the ethics of care. Feminist considerations on justice, morality and politics, Routledge, London.

Sevilla-Sanz, A., Gimenez-Nadal, J. I., Fernández, C., 2010, “Gender roles and the division of unpaid work in Spanish households", en Feminist Economics, 16 (4), pp. 137-184.

Sullivan, O., 2004, "Changing gender practices within the household: a theoretical perspective", en Gender and Society, 18 (2), pp. 207-222.

Taylor, R. F., 2004, "Extending conceptual boundaries: Work, voluntary work and employment", en Work, Employment $\in$ Society, 18 (1) (March 01), pp. 29-49. 
Taylor, R. F., 2005, "Rethinking voluntary work", en The Sociological Review, 53, pp. 117-135.

Torns, T., 2005, "De la imposible conciliación a los permanentes malos arreglos", en Cuadernos de Relaciones Laborales 23 (1), pp. 15-33.

Torns, T., 2007, "El cuidado de la dependencia. Un trabajo de cuidado", en Mientras tanto, 13, pp. 33-43.

Torns, T., Borràs, V., Carrasquer, P., Moreno, S., Castelló, L., Grau, A., 2011, "Trayectorias laborales y de vida. Una aproximación al modelo de empleo español", Working Paper $N^{\circ} 17$, en QUIT, Universitat Autónoma de Barcelona, Barcelona.

Torns, T., Carrasquer, P., Parella, S., Recio, C., 2007, "Les dones al mercat de treball a catalunya", en T. Torns (Ed.), Les dones i el treball a Catalunya: mites i certeses, Intitut Catalá de les Dones, Barcelona, pp. 28-53.

Tronto, J., 1987, "Más allá de la diferencia de género. Hacia una teoría del cuidado", en Signs: Journal of Women in Culture and Society, vol. 12, pp. 1-17 [Traducción del programa de democratización de las relaciones sociales. Escuela de posgrado. Universidad Nacional de General San Martín. Aprobada y autorizada su publicación por la autora].

Vega, C., 2009, Culturas del cuidado en transición. Espacios, sujetos e imaginarios en una sociedad de migración, Editorial UOC, Barcelona.

West, C., Zimmerman, D. H., 1987, "Doing gender", en Gender and Society, 1 (2), pp. 125-151.

Young, I. M., 1990, "Imparcialidad y lo cívico público. Algunas implicaciones de las críticas feministas a la teoría moral y política", en S. Benhabib y D. Cornell (Eds.), Teoría feminista y teoría crítica, Edicions Alfons el Magnànim, Valencia, pp. 89-118. 